\title{
Delayed Onset Tumor Lysis Syndiome after Initial Pomalidomide Treatment in a Patient with Refractory Multiple Myeloma
}

Takaaki Maekawa, Junya Take, Toshikuni Kawamura, Toshikatsu Horiuchi, Shoichiro Kato, Reina Saga, Takeshi Yamamura, Junichi Watanabe, Ayako Kobayashi, Shinichi Kobayashi, Ken Sato, Fumihiko Kimura

Division of Hematology, Department of Internal Medicine, National Defense Medical College, Namiki 3-2, Tokorozawa, Saitama, Japan.

\section{Abstract:}

An 82-year-old male with multiple myeloma developed tumor lysis syndrome (TLS) 9 days after initial pomalidomide and dexamethasone treatment (PomD). His prior therapy included melphalan and prednisolone; bortezomib and dexamethasone; and lenalidomide and dexamethasone without any TLS symptoms. PomD was administered due to myeloma progression, and his renal function improved gradually without acute adverse effects. However, on day 9, his renal function suddenly worsened, and serum lactate dehydrogenase, uric acid, potassium, and phosphorous levels were elevated. There were no symptoms of infection, or remarkable changes in urinary findings. After PomD was discontinued and hydration was started on the same day, his renal function improved immediately, and treatment was resumed on day 24 . TLS is a relatively rare, but life-threatening, complication in multiple myeloma patients and this onset period is sometimes quite different from other hematological malignancies. In addition to myelosuppression, TLS is a critical adverse event of PomD that may have delayed onset.

Key words: Tumor Lysis Syndrome, Pomalidomide, Immunomodulatory Drug, Adverse Effects, Multiple Myeloma.

\section{Introduction}

Tumor lysis syndrome (TLS) is a life-threatening complication in the treatment of malignancies. TLS was previously considered extremely rare $(\sim 1 \%)$ in multiple myeloma (MM) patients [1], but several reports have been published after the introduction of new agents [2-12]. Hyperproliferative diseases in bone marrow, circulating plasmablasts, and $13 q$ deletion have been identified as risk factors for TLS in MM [1]. Pomalidomide (Pom) is an immunomodulatory drug recently used for the treatment of relapsed/refractory $M M$, with encouraging results [13]. Adverse events observed in Pom treatment are similar to those of lenalidomide, and the most common grade 3-4 adverse event associated with Pom is myelosuppression [14]. In lenalidomide treatment, only 3 cases of TLS in

\section{Corresponding Author: Dr. Takaaki Maekawa}

Email: dri3011@ndmc.ac.jp

Received: November 4, 2015 | Accepted: January 11, 2016 | Published Online: February 10, 2016

This is an Open Access article distributed under the terms of the Creative Commons Attribution License (creativecommons.org/licenses/by/3.0)

Conflict of interest: None declared | Source of funding: Nil | DOl: http://dx.doi.org/10.17659/01.2016.0015 
MM have been reported without details $[17,18]$. Although only one case of TLS after Pom treatment was observed in a phase 2 study (MM-002), a postmarketing surveillance in Japan by the Celgene Corporation identified 7 cases of TLS, including our case; however, apart from two cases, further details were not disclosed. We present here a patient with refractory $M M$ who developed delayed onset TLS during the first cycle of treatment with Pom.

\section{Case Report}

An 82-year-old male was diagnosed with $\lg G \kappa$ asymptomatic MM, ISS stage 1 , in 2005. Because of the gradual increase of $M$ protein, he received melphalan and prednisolone chemotherapy followed by bortezomib and dexamethasone treatment in 2010. Despite its effectiveness, bortezomib was stopped due to emerging lung injury after the first administration. Chromosomal abnormalities of p53 and $13 q$ deletion were detected in April 2011 . The patient then received 46 cycles of lenalidomide and dexamethasone treatment from May 2011 to May 2015, and his best response was VGPR. However, light chain escape developed, and the $\kappa / \lambda$ rate of free light chains remarkably increased concomitant with gradual progression of renal impairment from October 2014. Increasing the dosage of lenalidomide and combined use of clarithromycin were unable to stop MM progression.

We started pomalidomide and dexamethasone treatment (PomD) in June 2015. The pretreatment serum biochemical profile showed creatinine $1.68 \mathrm{mg} / \mathrm{dL}$, uric acid $3.6 \mathrm{mg} /$ $\mathrm{dL}$, potassium $4.6 \mathrm{mEq} / \mathrm{L}$, calcium $8.4 \mathrm{mg} / \mathrm{dL}$, phosphorus $2.9 \mathrm{mg} / \mathrm{dL}$, and lactate dehydrogenase (LDH) $403 \mathrm{IU} / \mathrm{L}$. Bone marrow aspiration was not performed due to the patient's wishes. He was treated with $4 \mathrm{mg} /$ day Pom and $20 \mathrm{mg} /$ week dexamethasone together with $1000 \mathrm{ml} /$ day of hydration and no antihyperuricemic agent. Clarithromycin, sulfamethoxazole/trimethoprim, and fluconazole were administered for infection prophylaxis. His renal function improved gradually without acute adverse effects. However, on day 9, his renal function suddenly worsened, and laboratory tests showed creatinine $2.24 \mathrm{mg} / \mathrm{dL}$, uric acid 9.9 $\mathrm{mg} / \mathrm{dL}$, potassium $4.9 \mathrm{mEq} / \mathrm{L}$, calcium $8.4 \mathrm{mg} / \mathrm{dL}$, phosphorus $5.4 \mathrm{mg} / \mathrm{dL}$, and LDH $529 \mathrm{IU} / \mathrm{L}$. His vital signs were stable, and only mild disorientation was observed at that time. There were no symptoms of infection and no remarkable changes in urine volume or urinary findings. Under the diagnosis of TLS, we discontinued Pom, clarithromycin, and fluconazole, and started $2000 \mathrm{ml} /$ day of hydration and furosemide to maintain high urine output and $20 \mathrm{mg} /$ day febuxostat for hyperuricemia. The treatments were effective; his renal dysfunction, hyperuricemia, and hyperphosphatemia almost completely improved by day 11 [Fig.1].

While TLS was controlled, cessation of Pom treatment was continued due to a platelet count decrease to $25,000 / \mu \mathrm{L}$ on day 11 . On day 24 , his platelet count recovered to $57,000 / \mu \mathrm{L}$, and Pom treatment was resumed at a reduced dosage of $3 \mathrm{mg} /$ day. No TLS symptoms were observed following this incident.

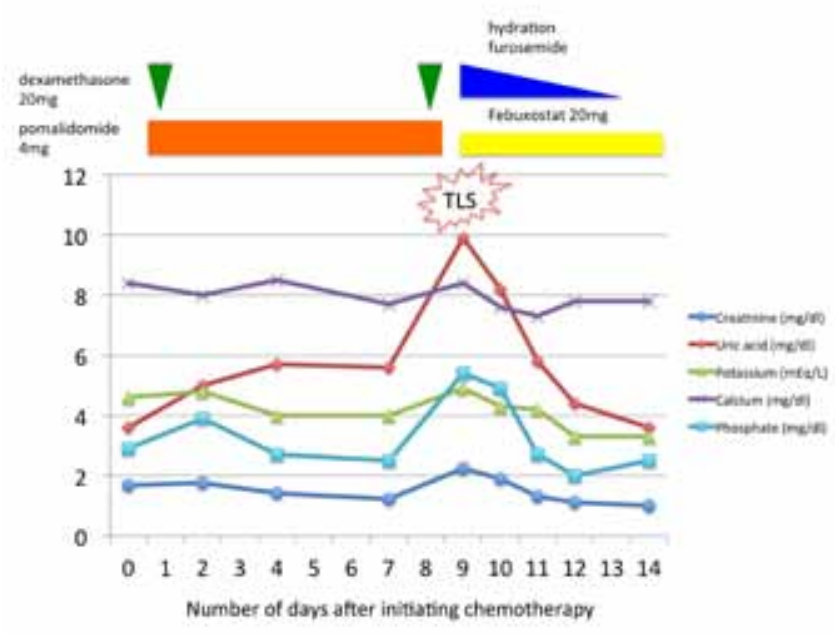

Fig.1: Serial changes in creatinine, uric acid, and electrolyte concentrations in the patient during the first cycle of treatment with Pom. 


\section{Discussion}

TLS can be classified into either laboratory type, defined as two or more of the following metabolic abnormalities (hyperuricemia, hyperkalemia, hyperphosphatemia, and hypocalcemia) occurring within 3 days before, or up to 7 days after the initiation of treatment, or clinical type TLS, defined as laboratory TLS accompanied by an increased creatinine level, seizures, cardiac dysrhythmia, or death [15]. The present case was diagnosed as clinical TLS with hyperuricemia, hyperphosphatemia, and increased creatinine level. Interestingly, TLS after Pom treatment does not always occur in the earlier period of administration, but has been reported to develop on day 29 (case MM-002) and day 15 (case of post-marketing surveillance in Japan by the Celgene Corporation) and day 9 (our case). This may be due to the relatively delayed onset of Pom activity. Our case improved immediately after treatment, but case MM-002 died 2 days after TLS and the other case also died. Despite the 7-day limitation included in the definition of TLS, clinicians must be aware of the possibility of delayed onset.

The incidence of TLS in MM was reported to be approximately $1 \%$ in 1999 [1], and $5 \%$ in Japanese patients treated with bortezomib (26 out of 525 cases) [16]. Sezer et al. reported that 7 of the 496 myeloma patients $(1.4 \%)$ treated with bortezomib in three, phase II, multi-center trials, developed TLS within 3 weeks of starting bortezomib therapy [2]. Six of these patients had a high tumor burden and four patients had 13q deletion. There have also been additional reports of TLS after bortezomib treatment [3-9], almost all of whom suffered from relapsed/refractory $M M$, and had been pretreated by several courses of treatment, including autologous stem cell transplantation.

In contrast, limited information of TLS after IMiDs treatment is available (Table 1). There have been three patients reported to have developed TLS during the first cycle of thalidomide treatment [10-12]. All had a high tumor burden, and one with $13 q$ deletion showed delayed onset of TLS. In lenalidomide treatment, post-marketing surveillance by the Celgene Corporation identified 13 cases of TLS $(0.5 \%)$ in 2671 Japanese MM patients. In a phase II study, 1 of the 21 newly diagnosed MM patients treated with lenalidomide, cyclophosphamide, and prednisone was reported to have developed TLS [17]. Baz et al. reported two TLS cases in 62 relapsed/refractory MM patients treated with lenalidomide and pegylated liposomal doxorubicin-based chemotherapy [18]. Unfortunately, there have been no detailed case reports on TLS after lenalidomide treatment. In Pom treatment, although only one case of TLS after Pom treatment was observed in a phase 2 study (MM002), a post-marketing surveillance in Japan by the Celgene Corporation identified 7 cases of TLS, including our case; however, apart from two cases, further details were not disclosed.

Table 1: Reports of TLS after treatment with IMiDs

\begin{tabular}{|c|c|c|c|c|c|c|c|c|}
\hline $\begin{array}{l}\text { Patient } \\
\text { number }\end{array}$ & $\begin{array}{l}\text { Age / } \\
\text { Gender }\end{array}$ & Agent & Dosage & $\begin{array}{l}\text { Tumor burden } \\
\text { aberration }\end{array}$ & Chromosome & Onset of TLS & Prognosis & Reference \\
\hline 1 & $71 / M$ & thalidomide & $100 \mathrm{mg} /$ day & $6 \%$ in $P B$ & $13 q$ deletion & day 13 & $\begin{array}{l}\text { maintenance } \\
\text { dialysis }\end{array}$ & 10 \\
\hline 2 & $59 / F$ & thalidomide & $200 \mathrm{mg} /$ day & $90 \%$ in $\mathrm{BM}$ & $\mathrm{n} / \mathrm{a}$ & day 6 & dead & 11 \\
\hline 3 & $60 / M$ & thalidomide & $200 \mathrm{mg} /$ day & $80 \%$ in $\mathrm{BM}$ & $\mathrm{n} / \mathrm{a}$ & day 3 & resume $\mathrm{Tx}$ & 12 \\
\hline
\end{tabular}

BM: bone marrow, PB: peripheral blood, $n$ /a: not available 
Incidence of TLS in MM may be more frequent in patients treated with bortezomib or combination chemotherapy compared with patients treated with an immunomodulatory drug alone; however, a high tumor burden and $13 q$ deletion may be risk factors of TLS in both patient groups. Chang et al. reported two cases of TLS in patients with light chain MM, and suggested that renal dysfunction may be risk factor of TLS in MM patients [19]. Our patient had a high tumor burden with renal dysfunction (the $\kappa / \lambda$ rate of free light chains was over the measurable amount at the beginning of PomD treatment) and $13 q$ deletion.

Concomitant use of Pom and clarithromycin may have been an additional factor contributing to the TLS in the present case. In our institution, new quinolones are usually used for infection prophylaxis, yet due to the renal dysfunction of the present case, clarithromycin was administered for infection prophylaxis. Clarithromycin inhibits CYP3A4, attenuating metabolization of Pom; elevated levels of Pom may be associated with the onset of TLS. At present, MM is regarded as having a low risk of TLS [20], yet MM patients with TLS risk factors treated with high-dose chemotherapy or new agents should be closely monitored. TLS should be treated with hydration and prophylactic administration of antihyperuricemic agents, and careful monitoring of electrolytes and renal function is important.

\section{Conclusion}

Pom is a new agent with encouraging results, but clinicians must be aware of the possibility of several adverse events. In addition to myelosuppression, TLS is a critical adverse event that may have delayed onset. The incidence of TLS in MM patients treated with Pom remains unclear at present, and further accumulation of cases is necessary.

\section{References}

1. Fassas $A B$, Desikan $K R$, Siegel $D$, Golper TA, Munshi NC, Barlogie B, et al. Tumor lysis syndrome complicating high-dose treatment in patients with multiple myeloma. Br J Haematol. 1999; 105:938-941.

2. Sezer $O$, Vesole DH, Singhal S, Richardson $P$, Stadtmaver E, Jakob C, etal. Bortezomib-induced tumor lysis syndrome in multiple myeloma. Clin lymphoma myeloma. 2006;7:233-235.

3. Berenson JR, Yang HH, Vescio RA, Nassir Y, Mapes $R$, et al. Safety and efficacy of bortezomib and melphalan combination in patients with relapsed or refractory multiple myeloma: updated results of a phase $1 / 2$ study after longer follow-up. Ann Hematol. 2008;87:623-631.

4. Jaskiewicz AD, Herrington JD, Wong L. Tumor lysis syndrome after bortezomib therapy for plasma cell leukemia. Pharmacotherapy. 2005; 25:1820-1825.

5. Terpos E, Politou M, Rahemtulla A. Tumor lysis syndrome in multiple myeloma after bortezomib (VELCADE) administration. J Cancer Res Clin Oncol. 2004; 130:623-625.

6. Kenealy MK, Prince HM, Honemann D. Tumor lysis syndrome early after treatment with bortezomib for multiple myeloma. Pharmacotherapy. 2006;26:1 205-1 206: discussion 6.

7. Tanimura A. Tumor lysis syndrome after bortezomib treatment in refractory/relapsed myeloma patients. Haematologica. 2011;96 (suppl 1):S130. Abstract P344.

8. Furtado $M$, Rule $S$. Bortezomib-associated tumor lysis syndrome in multiple myeloma. Leuk Lymphoma. 2008;49:2380-2382.

9. Chim CS. Rapid complete remission in multiple myeloma with bortezomib/thalidomide/ dexamethasone combination therapy following development of tumor lysis syndrome. Cancer Chemother Pharmacol. 2008;62:181-182. 
10. Huston A, Brown J, Roodman GD. Tumor lysis syndrome following thalidomide and dexamethasone therapy for newly diagnosed multiple myeloma. Exp Hematol. 2006;34:1616.

11. Cany L, Fitoussi O, Boiron JM, Marit G, et al. Tumor lysis syndrome at the beginning of thalidomide therapy for multiple myeloma. J Clin Oncol. 2002;20:2212.

12. Fuente N, Mane JM, Barcelo R, Munoz A, PerezHoyos T, Lopez-Vivanco $G$, et al. Tumor lysis syndrome in a multiple myeloma treated with thalidomide. Ann Oncol. 2004;1 5:537.

13. San Miguel J, Weisel K, Moreau $P$, Lacy $M$, Song $K$, Delforge $M$, et al. Pomalidomide plus low-dose dexamethasone versus high-dose dexamethasone alone for patients with relapsed and refractory multiple myeloma (MM-003): a randomised, open-label, phase 3 trial. Lancet Oncol. 2013;14(11):1055-1066.

14. Dimopoulos MA, Leleu $X$, Palumbo A, Moreau $P$, Delforge $M$, Cavo $M$, et al. Expert panel consensus statement on the optimal use of pomalidomide in relapsed and refractory multiple myeloma. Leukemia. 2014;28:15731585.

15. Howard SC, Jones DP, Pui CH. The tumor lysis syndrome. N Engl J Med. 2011;364:18441854.
16. lida S, Mukai HY, Ohyashiki, et al. Postmarketing surveillance of bortezomib treatment for multiple myeloma (MM) in Japan: result of interim analysis by the Velcade Japan Proper Use Guideline Committee. Blood (ASH Annual Meeting Abstracts). 2008;1 12: Abstract 5198.

17. Suvannasankha A, Farag S, O'Bryant RA, et al. Phase 2 clinical trial of oral lenalidomide, cyclophosphamide, and prednisone (RCP) for newly diagnosed multiple myeloma patients. Blood (ASH Annual Meeting Abstracts). 2009;1 14: Abstract 2882.

18. Baz R, Walker E, Karam MA, Choveiri TK, Jawde RA, Bruening $K$, et al. Lenaidomide and pegylated liposomal doxorubicin-based chemotherapy for relapsed or refractory multiple myeloma: safety and efficacy. Ann Oncol. 2006;17(12):1766-1771.

19. Chang H, Lee SY, Tang TC. Tumor lysis syndrome in patients with light chain multiple myeloma: report of two cases. Chang Gung Med J. $2011 ; 34$ (suppl 6): 70-75.

20. Cairo MS, Coiffier B, Reiter A, Younes A, et al. Recommendation for the evaluation of risk and prophylaxis of tumor lysis syndrome (TLS) in adults and children with malignant diseases: an expert TLS panel consensus. Br J Haematol. 2010;1 49:578-586. 